\title{
COMPLICAÇÕES ORAIS EM PACIENTES TRATADOS COM RADIOTERAPIA OU QUIMIOTERAPIA EM UM HOSPITAL DE SANTA CATARINA.
}

\section{ORAL COMPLICATIONS IN PATIENTS TREATED WITH RADIOTHERAPY OR CHEMOTHERAPY IN A HOSPITAL IN SANTA CATARINA.}

\author{
Deivid de Freitas Floriano* \\ Patricia Fernandes Avila Ribeiro** \\ Angela Catarina Maragno"** \\ Karoline Rossi" ${ }^{\cdots *}$ \\ Priscyla Waleska Targino de Azevedo Simões**.***
}

\begin{abstract}
RESUMO
Objetivo: Este estudo se propôs a avaliar as complicações orais em pacientes tratados com radioterapia ou quimioterapia. Introdução: Mesmo que a radioterapia e a quimioterapia apresentem diminuição da taxa de mortalidade associada ao câncer, a morbidade relacionada ao tratamento pode levar a complicações sistêmicas importantes como, por exemplo, na região bucomaxilofacial, o que faz aumentar o tempo de internação e os custos do tratamento, bem como afetar diretamente a qualidade de vida dos pacientes oncológicos. Métodos: Foi realizado um estudo transversal, descritivo, observacional e de campo com uma amostra (por conveniência), composta por 96 pacientes, maiores de 18 anos, que estavam sob tratamento antineoplásico, no ambulatório de oncologia (radioterapia e quimioterapia) em um hospital de Santa Catarina. O estudo foi realizado nos meses de setembro e outubro de 2016. Os resultados indicaram a predominância de pacientes do gênero feminino, com o sistema reprodutor como o sítio oncológico mais encontrado, em maior proporção na idade de 56 a 65 anos, tratados com quimioterapia. Resultados: Foram encontrados 06 (seis) tipos de lesões, alterações ou patologias em $90 \%$ dos pacientes, destacando-se a xerostomia, a mucosite e a candidíase. Conclusões: A xerostomia, a mucosite e a candidíase foram as lesões mais encontradas nos pacientes. Tais problemas podem ser decorrentes da falta de tratamento odontológico antes da terapia oncológica, bem como durante o tratamento.
\end{abstract}

Descritores: Odontologia · Câncer · Antineoplásicos.

\section{ABSTRACT}

Objective: This study aimed to evaluate oral complications in patients treated with radiotherapy or chemotherapy. Introduction: Although radiotherapy and chemotherapy have a reduction in cancer-associated mortality, treatment-related morbidity can lead to important systemic complications, such as in the mouth-maxillofacial region, which hospitalization and the costs of treatment, as well as directly affect the quality of life of cancer patients. Methods: A cross-sectional, descriptive, observational and field study was carried out with a sample (for convenience), composed of 96 patients, older than 18 years, who underwent antineoplastic treatment, at the oncology clinic (radiotherapy and chemotherapy) in a hospital of Santa Catarina. The study was conducted in September and October 2016. The results indicated the predominance of female patients, with the reproductive system as the most commonly found cancer site, at a greater proportion at age from 56 to 65 years, treated with chemotherapy. Results: Six (6) types of lesions, alterations or pathologies were found in $90 \%$ of the patients, with xerostomia, mucositis and candidiasis. Conclusions: Xerostomia, mucositis and candidiasis were the most frequent lesions in patients. Such problems may be due to the lack of dental treatment prior to oncologic therapy, as well as during this treatment.

Descriptors: Dentistry · Cancer · Antineoplastic agents.

* Graduado em Odontologia - Universidade do Extremo Sul Catarinense. Email: deividff@hotmail.com.

** Mestre em Patologia Bucal - Faculdade de Odontologia São Leopoldo Mandic. Docente do Curso de Odontologia - Universidade do Extremo Sul Catarinense. Email: patricia@precisao.org.

*** Especialista em Radiologia Odontológica - Universidade Federal de Santa Catarina. Docente do Curso de Odontologia - Universidade do Extremo Sul Catarinense. Email: acmaragno@gmail.com.

**** Graduada em Odontologia - Universidade do Extremo Sul Catarinense. Email: karoline_rossi@hotmail.com

***** Doutora em Ciências da Saúde - Universidade do Extremo Sul Catarinense. Docente do Curso de Odontologia - Universidade do Extremo Sul Catarinense. Email: pri@unesc.net. 


\section{N T RO DUÇÃO}

Neoplasia é o nome dado a mais de 100 doenças que têm como principal característica o crescimento desordenado (maligno/benigno) de células, capazes de invadir tecidos e órgãos vizinhos ou distantes (metástases) por meio da corrente sanguínea e do sistema linfático ${ }^{1,2}$. Essas células dividem-se rapidamente, sendo muito agressivas e incontroláveis, e formam uma massa celular chamada tumor ${ }^{3}$. Os tumores representam o acúmulo de células cancerosas, sendo também denominados de neoplasias malignas ${ }^{1,2,3}$. Quando o tumor tem início em tecidos epiteliais como pele ou mucosas ele é chamado de carcinoma. Já quando começa em tecidos conjuntivos como osso, músculo ou cartilagem é denominado de sarcoma ${ }^{1}$.

A diversidade de tipos de tumores é muito grande, com causas multifatoriais. Mas, em geral, uma junção de fatores genéticos e agentes externos contribui para o seu aparecimento ${ }^{3}$, desencadeando o mau funcionamento de genes que controlam o crescimento, a divisão e a maturação celular².

O câncer responde pela segunda causa de mortes por doença no mundo ${ }^{2,4,5}$, sendo precedido apenas por patologias cardiovasculares ${ }^{5}$. De acordo com dados do relatório da Agência Internacional para Pesquisa em Câncer da Organização Mundial da Saúde (IARC/OMS), a incidência global de câncer duplicou nos últimos 30 anos $^{3}$.

No Brasil, estimativas apontam que haverá 596.070 novos casos de neoplasias em 2016, dos quais 295.200 em homens, e 300.870 entre as mulheres. O mais incidente é o carcinoma de pele não melanoma, em ambos os gêneros. Depois deste, para os homens, os tumores mais encontrados são os de próstata, pulmão, cólon e reto, estômago, cavidade oral, esôfago, bexiga, laringe e leucemias. Entre as muIheres, as maiores incidências serão de cânceres de mama, cólon e reto, colo do útero, pulmão, estômago, corpo do útero, ovário e glândula tireoide ${ }^{6}$.

A escolha do método de tratamento depende da natureza e extensão do tumor ${ }^{7}$. A cirurgia (ressecção), a radioterapia e a quimioterapia são as opções mais frequentes para o manejo da doença oncológica ${ }^{8,9}$.

Estima-se que aproximadamente $40 \%$ dos pacientes que recebem radioterapia e quimioterapia apresentarão complicações orais decorrentes de estomatotoxicidade direta ou indireta ${ }^{4}$. Problemas na boca mais frequentemente resultam de radioterapia, mas a cirurgia e a quimioterapia também podem causar ou agravar problemas dentários e periodontais ${ }^{10}$.

Diante disso, a correta compreensão desses sinais e sua correlação com sintomas e drogas ou radiação utilizadas nos tratamentos oncológicos tornam esses tipos de manifestações mais previsíveis, o que facilita a prevenção e o tratamento, sendo de grande importância a integração da odontologia na equipe médica de oncologia.

Com base nessas considerações, este estudo tem por objetivo avaliar complicações orais em pacientes tratados com radioterapia ou quimioterapia em um hospital de Santa Catarina.

\section{METODOLOG I A}

Trata-se de um estudo transversal, descritivo e observacional, com uma amostra (por conveniência), composta por 96 pacientes, maiores de 18 anos, que estavam sob tratamento antineoplásico, no ambulatório de oncologia (radioterapia e quimioterapia) em um hospital de Santa Catarina. Pacientes oncológicos submetidos apenas à cirurgia ou tratados com outras terapias associadas (imunoterapia, transplante de medula óssea, entre outras) foram excluídos da pesquisa.

Foi realizada uma pesquisa de campo, com coleta de dados junto aos pacientes da amostra nos meses de setembro e outubro de 2016, bem como exame clínico oral dos mesmos e aplicação de questionário feito pelo pesquisador. As variáveis foram: perfil do paciente (gênero, idade, elitismo, tabagismo, sítio neoplásico, tratamento oncológico/combinação de tratamento); análise clínica das alterações/ lesões na região bucomaxilar; tratamento odontológico anterior ao tratamento antineoplásico; tratamento ou acompanhamento odontológico junto com o tra-
FLORIANO DF RIBEIRO PFA MARAGNO AC ROSSI K

SIMOEES PWTA

COMPLICAÇÕES ORAIS EM PACIENTES TRATADOS COM RADIOTERAPIA OU QUIMIOTERAPIA EM UM HOSPITAL DE SANTA CATARINA
REV. ODONTOL. UNIV, CID, SÃO PAULO $2017 ; 29(3)$ : $230-6$, SET - DEZ 
FLORIANO DF

RIBEIRO PFA

MARAGNO AC

ROSSI K

SIMÕES PWTA

COMPLICAÇÕES ORAIS EM PACIENTES TRATADOS COM RADIOTERAPIA OU QUIMIOTERAPIA EM UM HOSPITAL DE SANTA CATARINA

\section{2}

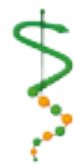

REV, ODONTOL

UNIV. CID, SÃO

PAULO

$2017 ; 29(3)$ :

230-6, SET - DEZ tamento da neoplasia e pesquisa em prontuários.

O exame clínico foi realizado por um profissional, cirurgião-dentista, e por um acadêmico de odontologia. O exame foi realizado no leito de internação do paciente, consistindo da anamnese e do exame físico e clínico intrabucal, sob luz artificial, de forma clínico-visual não invasiva, com espátula de madeira, gaze e uso de luvas de procedimento, descartadas após o exame de cada paciente. O exame clínico intrabucal possibilitou verificar as alterações/lesões presentes nos pacientes da amostra. Utilizou-se um questionário com perguntas objetivas e subjetivas direcionadas ao paciente e preenchidas pelo pesquisador; foram anotadas informações sobre: etilismo; tabagismo; combinação de tratamento (radioterapia/quimioterapia/ ambas); se realizou tratamento odontológico anterior ao tratamento antineoplásico; e se, junto ao tratamento oncológico, realizou algum tipo de tratamento ou acompanhamento odontológico.

Após a coleta de dados, foi elaborado um banco de dados no software Statistical Package for the Social Sciences (SPSS) versão 20, aplicativo também utilizado para a análise estatística. Os resultados foram tratados por meio de estatísticas simples, para se observar a ocorrência $(\mathrm{N})$ e a prevalência (\%). Os resultados foram representados por meio de tabelas e tratados com abordagem quantitativa.

O projeto foi aprovado pelo Comitê de Ética da Universidade do Extremo Sul Catarinense - UNESC sob o parecer №: 1.561.488. Como também pelo Comitê de Ética da instituição hospitalar sob o parecer $\mathrm{n}^{\mathrm{o}}$ 1.726.698.

Visando atender ao disposto na Res. no466/12 do Conselho Nacional de Saúde, foi entregue a cada paciente incluído na amostra um Termo de Consentimento Livre e Esclarecido (TCLE), em duas vias impressas, que foi assinado pelo participante e pesquisador e entregue uma via ao entrevistado e uma para o pesquisador.

\section{RESULTADOS}

Em relação ao perfil dos pacientes, foram verificadas as variáveis relacionadas ao gênero, idade, cor, nacionalidade, eli- tismo e tabagismo (Tabela 1).

Identifica-se a predominância de $55,2 \%$ de pacientes do gênero feminino $(n=53)$, porém, muito próximos foram encontrados $44,8 \%$ do gênero masculino $(n=43)$. Em relação à idade, foram identificados pacientes oncológicos em todas as faixas etárias investigadas ( $<18$ anos), com expressiva prevalência $(35,4 \%)$ para a idade situada entre 56 a 65 anos $(n=35)$. Os resultados também indicaram que não há prevalência de etilismo, com índice de $95,8 \%$ dos casos $(n=92)$. Da mesma forma, não foram registrados casos prevalentes de tabagismo nos pacientes, 79,2\% $(n=79)$.

Em relação aos aspectos neoplásicos dos pacientes da amostra (Tabela 2), verifica-se que prevalece o sítio do aparelho reprodutor de localização da neoplasia, com 36,1\% (n=35). A quimioterapia é a terapia mais referida quanto ao tratamento, com $62,5 \%$ dos casos $(n=60)$.

Conforme anamnese odontológica (Tabela 3), foram encontrados 06 (seis) tipos de lesões, alterações ou patologias em 87 pacientes da amostra, ou seja, em $90 \%$ do total. Entre as lesões mais evidenciadas, destacou-se a xerostomia, com 71,9\% ( $n=69)$, seguida da mucosite, com $67,7 \%$ $(n=65)$, e em terceiro lugar a candidíase, com $32,3 \%(n=31)$.

Ao se avaliar a realização de tratamento odontológico, tanto anterior à terapia neoplásica como durante a terapia oncológica (Tabela 4), pôde-se constatar que os pacientes não se submeteram a nenhum tipo de tratamento ou acompanhamento odontológico, ocorrido em $77,1 \%$ $(n=74)$. Além disso, durante o tratamento antineoplásico, evidencia-se que a totalidade da amostra não recebe nenhum tipo de tratamento ou acompanhamento odontológico.

\section{I SCUSSÃO}

O objetivo principal neste estudo foi avaliar as complicações orais em pacientes tratados com radioterapia ou quimioterapia. Com uma amostra de 96 pacientes oncológicos de um hospital de grande porte no Estado de Santa Catarina, observou-se a predominância de $55,2 \%$ de pacientes do gênero feminino. Além disso, 
Tabela 1 - Perfil dos Pacientes

\begin{tabular}{|c|c|c|c|}
\hline \multicolumn{4}{|l|}{ VARIÁVEL } \\
\hline Sexo & \multicolumn{2}{|c|}{ № } & $\%$ \\
\hline Masculino & \multicolumn{2}{|c|}{43} & 44,8 \\
\hline Feminino & \multicolumn{2}{|c|}{53} & 55,2 \\
\hline TOTAL & \multicolumn{2}{|c|}{96} & 100,0 \\
\hline \multicolumn{4}{|l|}{ VARIÁVEL } \\
\hline Idade & \multicolumn{2}{|c|}{ № } & $\%$ \\
\hline 18 a 25 & \multicolumn{2}{|c|}{02} & 2,0 \\
\hline 26 a 35 & \multicolumn{2}{|c|}{05} & 5,2 \\
\hline 36 a 45 & \multicolumn{2}{|c|}{09} & 9,3 \\
\hline 46 a 55 & \multicolumn{2}{|c|}{18} & 19,0 \\
\hline 56 a 65 & \multicolumn{2}{|c|}{35} & 35,4 \\
\hline 66 a 75 & \multicolumn{2}{|c|}{17} & 18,0 \\
\hline 76 ou mais & \multicolumn{2}{|c|}{11} & 11,1 \\
\hline TOTAL & \multicolumn{2}{|c|}{96} & 100,0 \\
\hline VARIÁVEL & & & \\
\hline Etilista & № & & $\%$ \\
\hline Sim & 04 & & 4,2 \\
\hline Não & 92 & & 95,8 \\
\hline TOTAL & 96 & & 100,0 \\
\hline VARIÁVEL & & & \\
\hline Tabagista & № & & $\%$ \\
\hline $\operatorname{Sim}$ & 20 & & 20,8 \\
\hline Não & 76 & & 79,2 \\
\hline TOTAL & 96 & & 100,0 \\
\hline Fonte: Dados da Pesquisa & & & \\
\hline Tabela 2 - Aspectos Neoplásico & & & \\
\hline VARIÁVEL & & & \\
\hline Sítio & & № & $\%$ \\
\hline Cabeça e Pescoço & & 22 & 22,9 \\
\hline Reprodutor & & 35 & 36,1 \\
\hline Digestório & & 19 & 19,9 \\
\hline Outros & & 20 & 21,1 \\
\hline TOTAL & & 96 & 100,0 \\
\hline VARIÁVEL & & & \\
\hline Tratamento & & № & $\%$ \\
\hline Quimioterapia & & 60 & 62,5 \\
\hline Radioterapia & & 20 & 20,8 \\
\hline Quimioterapia e Radioterapia & & 16 & 16,7 \\
\hline TOTAL & & 96 & 100,0 \\
\hline
\end{tabular}

FLORIANO DF

RIBEIRO PFA

MARAGNO AC

ROSSI K

SIMÕES PWTA

COMPLICAÇÕES

ORAIS EM PACIEN-

TES TRATADOS COM

RADIOTERAPIA OU

QUIMIOTERAPIA EM

UM HOSPITAL DE

SANTA CATARINA

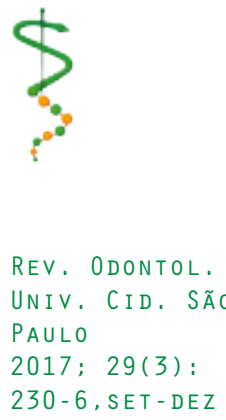


FLORIANO DF

RIBEIRO PFA

MARAGNO AC

ROSSI K

SIMOEES PWTA

COMPLICAÇÕES ORAIS EM PACIENTES TRATADOS COM RADIOTERAPIA OU QUIMIOTERAPIA EM UM HOSPITAL DE SANTA CATARINA

Tabela 3 - Alterações/Lesões/Patologias

\begin{tabular}{lll}
\hline \hline VARIÁVEL & & \\
Alterações & № & $\%$ \\
\hline Candidíase & 31 & 32,3 \\
Mucosite & 65 & 67,7 \\
Úlcera traumática & --- & --- \\
Xerostomia & 69 & 71,9 \\
Ardência bucal & 25 & 26,0 \\
Osteonecrose & --- & -- \\
Queilite angular & 17 & 17,7 \\
Pré-lesão & 8 & 8,3 \\
\hline \hline
\end{tabular}

Fonte: Dados da Pesquisa

Tabela 4 - Tratamento Odontológico

\begin{tabular}{lll}
\hline \hline VARIÁVEL & & \\
Tratamento Anterior à Oncoterapia & № & $\%$ \\
\hline Sim & 22 & 22,9 \\
Não & 74 & 77,1 \\
TOTAL & 96 & 100,0 \\
VARIÁVEL & & \\
Tratamento Durante à Oncoterapia & № & $\%$ \\
Sim & -- & -- \\
Não & 96 & 100 \\
TOTAL & 96 & 100,0 \\
\hline \hline
\end{tabular}

Fonte: Dados da Pesquisa

foi evidenciado que o sítio oncológico mais encontrado neste estudo foi o sistema reprodutor. Esse resultado corrobora com as estatísticas mundiais e brasileiras, as quais demonstram que o câncer mais prevalente nas mulheres é o de mama e colo de útero e dos homens o câncer de próstata ${ }^{1,6}$.

Segundo a amostra, o etilismo e o tabagismo parecem não estar associados às causas de neoplasia, tendo em vista que ambos os fatores foram recorrentes em menor número na amostra. Nesse sentido, sabe-se que, entre as causas para o desenvolvimento ou agravamento de neoplasias, incluem-se tabaco, álcool, inalação de produtos químicos, energia solar e radiação ionizante, microorganismos infecciosos, ambientes poluentes, medicamentos e até nutrientes, tidos como causas externas. As causas internas incluem envelhecimento, hereditariedade, condições imunes e erros no metabolismo ${ }^{1,2,6}$.

$\mathrm{Na}$ amostra, foram encontrados pacientes em maior proporção na idade de 56 a 65 anos, o que pode ser um dos fa- tores desencadeantes da doença. Isso porque o câncer, na terceira idade, tem uma incidência 11 (onze) vezes maior dentro dessa faixa etária, em comparação aos mais jovens ${ }^{6}$, embora outros motivos para a causa mereçam ser melhor investigados na amostra.

No que se refere à terapia antineoplásica, evidenciou-se a quimioterapia como tratamento de eleição, que ocorre em mais da metade da amostra, com $62,5 \%$, embora também ocorra pacientes tratados com radioterapia e outros com ambas as terapias ${ }^{11}$. A escolha do método de tratamento depende da natureza e extensão do tumor $^{7}$. A quimioterapia e a radioterapia são as opções mais frequentes para o manejo da doença oncológica ${ }^{8,9}$. Tais terapias podem ser realizadas sozinhas ou em combinação com outras modalidades ${ }^{11}$, 12. Contudo, a radioterapia pode causar danos irreversíveis para as glândulas salivares, cárie de radiação e osteorradionecrose, já a quimioterapia pode afetar o organismo local e sistêmico e causar grandes sequelas ${ }^{9,12}$. 
Confirmando esses achados, na amostra foram encontrados 06 (seis) tipos de lesões, alterações ou patologias em $90 \%$ dos pacientes, destacando-se a xerostomia, com $71,9 \%$, a mucosite, com $67,7 \%$ e a candidíase com 32,3\%.

A xerostomia, ou boca seca, pode ser um sintoma associado à baixa ou nenhuma produção de saliva pelas glândulas salivares. Pode ser provocada por fatores diversos, como diabetes mellitus ou também ser efeito colateral de certos medicamentos, como a radioterapia na região da cabeça e pescoço e a quimioterapia. Essa condição passaria a agravar todas as demais pelo papel primordial que a saliva exerce na proteção tecidual, mastigação e fonação ${ }^{13}$. Quando essa manifestação ocorre por consequência da quimioterapia, torna-se uma alteração transitória no funcionamento das glândulas salivares, cessando logo após o término do tratamento $^{14}$. Com a radioterapia, ocorre dano ou perda de ácinos das glândulas salivares maiores, resultando, muitas vezes, em severas e irreversíveis formas de disfunção salivar. Para minimizar o desconforto causado pela xerostomia, pode-se utilizar a saliva artificial, e também é importante orientar o paciente a consumir uma quantia maior de água ${ }^{15}$. A falta da saliva promove, o acúmulo de placa bacteriana e biofilme dental, aumentando a susceptibilidade da doença cárie, por isso é de fundamental importância consulta periódica ao dentista ${ }^{2}$.

Ja no caso da mucosite que se caracteriza por eritema e edema na mucosa, seguidos comumente de ulceração e descamação, é um problema clínico de difícil controle, podendo comprometer a deglutição, perda de paladar, a higiene oral e a capacidade de comunicação do paciente e pode resultar em ulcerações, muitas vezes obrigando à interrupção da radioterapia com evidente prejuízo ao tratamento. Bochechos com clorexidina a $0,12 \%$, podendo estar associada a hidróxido de alumínio, anti-inflamatórios, analgésicos, anestésicos tópicos, laserterapia de baixa potência, aplicação de chá de camomila, têm sido relatados na prevenção e controle da mucosite ${ }^{14,15}$.

A candidíase, por sua vez, pode favo- recer a propagação de microorganismos para outros órgãos e sistemas ${ }^{7}$. A radioterapia altera seletivamente a microflora oral, aumentando a colonização por cândida durante o tratamento. Também a quimioterapia com drogas citotóxicas e imunossupressivas irá aumentar a colonização por cândida ${ }^{15}$. A prevenção e o controle das infecções fúngicas são realizados através da utilização de antifúngicos locais ou sistêmicos, além de bochechos com suspensão oral ${ }^{4,15}$.

Tais problemas podem ser decorrentes da falta de tratamento odontológico antes da terapia oncológica, bem como durante esse tratamento ${ }^{13}$, tendo-se em vista que foi evidenciado que a maioria dos pacientes não se submeteram a esse tipo de conduta, em $77,1 \%$ do total da amostra, bem como foi verificado que a totalidade da amostra não recebe tratamento ou nenhum tipo de acompanhamento odontológico durante o tratamento antineoplásico.

Conforme se verifica, esse resultado se opõe à literatura, pois é consenso que a avaliação minuciosa, por parte do cirurgião-dentista, deve ser executada antes de se iniciar o tratamento oncológico, por meio de métodos radiográficos, exame clínico e anamnese completa ${ }^{16}$.

$\mathrm{Na}$ verdade, o cirurgião-dentista na equipe multiprofissional envolvido no tratamento da neoplasia pode impactar positivamente na qualidade de vida dos pacientes $^{9}$ ao prevenir e tratar problemas orais decorrentes das terapias oncológicas, por meio de um protocolo de atendimento odontológico que inclua medidas de condicionamento do meio bucal prévias à terapia, como profilaxia, remoção de cáries, tratamento periodontal e de focos periapicais, orientação para higiene oral e dieta ${ }^{7,12,14}$. Por isso, é importante a inserção do cirurgião-dentista na equipe multiprofissional para o diagnóstico precoce das manifestações bucais e acompanhamento no período de tratamento oncológico, tendo um trabalho em conjunto com a equipe multiprofissional, melhorando a qualidade de vida dos pacientes oncológicos.

$$
\text { CONCLUSÃO }
$$

FLORIANO DF RIBEIRO PFA MARAGNO AC ROSSI K

SIMÕES PWTA

COMPLICAÇÕES ORAIS EM PACIENTES TRATADOS COM RADIOTERAPIA OU QUIMIOTERAPIA EM UM HOSPITAL DE SANTA CATARINA

\section{5}

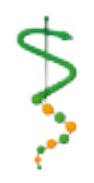

REV. OdONTOL. UnIV, CID, São PAULO 2017; 29(3): 230-6, SET-DEZ 
FLORIANO DF

RIBEIRO PFA

MARAGNO AC

ROSSI K

SIMÕES PWTA

COMPLICAÇÕES ORAIS EM PACIENTES TRATADOS COM RADIOTERAPIA OU QUIMIOTERAPIA EM UM HOSPITAL DE SANTA CATARINA

REV, ODONTOL UNIV, CID, SÃO PAULO $2017 ; 29(3):$ 230-6, SET - DEZ
Mediante os resultados apresentados, pode-se concluir que as principais complicações orais apresentadas pelos pacientes da amostra foram xerostomia, mucosite e candidíase.
Contudo, acredita-se que é possível melhorar a qualidade de vida antes, durante e após as terapias antineoplásicas, por meio de um protocolo odontológico.

\section{REFERÊNCIAS}

\section{REFERÊNCIAS}

1. Instituto Nacional Do Câncer. O que é o câncer? 2014 [Acesso em]; Disponível em: http://www1.inca.gov.br/conteudo view.asp?id=322.

2. Holtzclaw D, Toscano N, Marx R, Naylor G, Shumaker N, Richardson H, et al. Oral implications of cancer chemotherapy. JIACD Continuing Education 2009 1(5):51-69.

3. Pimentel OC. Entendendo o câncer. Porto Alegre: Artmed; 2014.

4. Hespanhol FL, Tinoco EMB, Teixeira HGC, Falabella MEV, Assis NMSP. Manifestações bucais em pacientes submetidos à quimioterapia. Ciênc saúde coletiva 2010 15(supl. 1):108594.

5. Wong HM. Oral complications and management strategies for patients undergoing cancer therapy. Scient World J 2014 2014(1):1-14.

6. Instituto Nacional Do Câncer. INCA estima que haverá 596.070 novos casos de câncer em 2016. 2015 [Acesso em]; Disponível em: http://www2.inca. gov.br/wps/wcm/connect/agencianoticias/ site/home/noticias/2015/estimativa_incidencia_cancer_2016.

7. Almeida RCCR. Perfil e condições bucais de pacientes oncológicos do hospital escola da universidade federal de Pelotas [Monografia de Especialização]. Pelotas: Universidade Federal de Pelotas; 2012.

8. Thanvi J, Bumb D. Impact of dental considerations on the quality of life of oral cancer patients. Indian journal of medical and paediatric oncology : official journal of Indian Society of Medical \& Paediatric Oncology 2014 Jan;35(1):66-70.
9. Leal VD, Ferreira LA, Santos MN, Figueiredo PTdS. Tratamento odontológico em pacientes oncológicos. Oral Sci 2012 4(2):37-42.

10. Jorewicz L. Managing dental complications in patients with head and neck cancer. Oncolog 2013 nov-dez;58(1):11-2.

11. Pozzobon JL, Ortiz FR, Braun K, Unfer B. Complicações bucais dos tratamentos de câncer de cabeça e pescoço e de malignidades hematológicas. RFO, Passo Fundo 2011 set/dez ;16(3):3426.

12. Jawad H, Hodson NA, Nixon PJ. A review of dental treatment of head and neck cancer patients, before, during and after radiotherapy: part 1 . British dental journal 2015 Jan;218(2):65-8.

13. Gaetti-Jardim Júnior $E$, Crivelini $M M$, Okamoto AC, Buso-Ramos MM, Ciesielski FIN, Sousa FRN, et al. Efeitos da radioterapia sobre as condições bucais de pacientes oncológicos. RPG rev pos-grad 2011 abr-jun;18(2):96-101.

14. Barillari ME, Goulart MN, Gomes ACP. Complicações das terapias antineoplásica: prevenção e tratamento da mucosite oral. Investigação 2015 14(6):121-4.

15. Paiva MDEB, Biase RdCCGd, Moraes JJdC, Ângelo AR, Honorato MCTdM. Complicaçõesoraisdecorrentesdaterapiaantineoplásica.ArquivosemOdontologia 2010 46(48-55.

16. Camargo AM. O tratamento pediátrico oncológico, suas complicações orofaciais e prevenção [Monografia de Especialização]. São Paulo: Associação Brasileira de Ensino Odontológico; 2001.

Recebido em 20/07/2017

Aceito em 14/08/2017 